\title{
More Powerful Test for Homogeneity of Means Under an Order Restriction in Time Series with Stationary Process
}

\author{
Abouzar Bazyari \\ Department of Statistics, Persian Gulf University, Bushehr, Iran
}

\begin{abstract}
Suppose that an order restriction is imposed among several means in time series. We are interested in testing the homogeneity of these unknown means under this restriction. In the present paper, a test based on the isotonic regression is done for monotonic ordered means in time series with stationary process and short range dependent sequences errors. A test statistic is proposed using the penalized likelihood ratio (PLR) approach. Since the asymptotic null distribution of test statistic is complicated, its critical values are computed by using Monte Carlo simulation method for some values of sample sizes at different significance levels. The power study of our test statistic is provided which is more powerful than that of the test proposed by Brillinger (1989). Finally, to show the application of the proposed test, it is applied to real dataset contains monthly Iran rainfall records.
\end{abstract}

Key words: Isotonic regression, Monte Carlo simulation, Penalized likelihood ratio, Test of trend

\section{Introduction}

In the application fields of statistical inference it is important to test stationary of the given time series. The interest centre on the stationary of mean, and the process of interest is assumed to be of the following form

$$
X_{k}=\theta_{k}+Z_{k},
$$

where the $\theta_{k}, k=0,1,2, \ldots$, are the means and $Z_{k}$ is a stationary process with mean 0 and finite covariances $\gamma(k)=\operatorname{cov}\left(Z_{i}, Z_{i+k}\right)=E\left(Z_{0} Z_{k}\right)$, for any value of $i$. For this plan, there has been both classical work on testing for the existence of a trend and more recent work on tests for an abrupt change. The present work falls between these two approaches in developing a test for a change, or trend that is monotonic but otherwise arbitrary. It is assumed that the homogeneity of means hypothesis as the null hypothesis of interest is

$$
H_{0}: \theta_{1}=\theta_{2}=\cdots=\theta_{n},
$$

against the alternative order hypothesis

$$
H_{1}: \theta_{1} \leq \theta_{2} \leq \cdots \leq \theta_{n}
$$


682 More Powerful Test for Homogeneity of Means Under an Order Restriction in Time Series with Stationary

Process

with at least one strict inequality in $H_{1}$. Darkhovsky (1994), shown that general change point problems can be reduced to the problem of testing the mean stability of some new sequences. The type of testing we consider is off-line, in that the data $\Omega=\Omega_{n}=\left(X_{1}, X_{2}, \ldots, X_{n}\right)$ have already been obtained before the analysis. The trend $\left\{\theta_{k}\right\}, k=0,1,2, \ldots$, is sometimes called the signal and the process $\left\{Z_{k}\right\}$ is the errors. The formulation is motivated in part by Iran rainfall data provided by a meteorologist from the Agricultural Experimental Station in Tehran. This dataset contains monthly rainfall records collected from 1898 to 2010. The meteorologist believes that there was a change in the mean, caused by the construction of a dam in Karaj from 1966 to 1976. Early work on the problem of testing mainly concerned the simple model in which the $\left\{Z_{k}\right\}, k=0,1,2, \ldots$, are independent and the $\left\{\theta_{k}\right\}$ only take two possible values. Then the alternative hypothesis has the following form

$$
H_{1}^{\prime}: \theta_{1}=\theta_{2}=\cdots=\theta_{v} \neq \theta_{v+1}=\cdots=\theta_{n},
$$

for some unknown change point $v$ such that $1 \leq v \leq n$. A statistical change point problem was first studied in the mid-1950s in the context of quality control in industrial processes. A change point is defined as a point in the time order when the probability distribution of a sequence of observations differs before and after that point. The literature of statistical change point has evolved over time and now includes a significant amount of scholarly work on change point analysis, and genetics, to name a few. The classical change point problem is to test for the existence of a change point and estimate its location if it exits. Picard (1985), presented a few techniques which may be useful in the analysis of time series when a failure is suspected. $\mathrm{He}$ presented two categories of tests and investigate their asymptotic properties: one, of nonparametric type, is intended to detect a general failure in spectrum; the other investigates the properties of likelihood ratio tests in parametric models which have a non-standard behaviour in this situation. Siegmund (1986) and Bhattacharya (1994), have written review articles, and Shaban (1980) has complied an annotated bibliography. In many literatures, the change-point problem for linear models has been discussed extensively. While most of the contributions are made from a posteriori point of view (we refer to, e.g., Bai, 1997, Csörgo and Horváth, 1997, and Perron, 2006), recently the sequential or on-line change-point detection has received more and more attention.

Chu et al. (1995) and Horváth et al. (2004), who suggested cumulative sum (CUSUM) procedures in different stochastic models. CUSUM procedures work best for relatively early changes but show a slower reaction the later the change occurs. Chu et al. (1995) investigated tests for structural change based on moving sums (MOSUMS) of recursive and least-squares residuals. They obtained the asymptotic critical values of the MOSUM test with recursive residuals and 
showed that the asymptotic critical values of the MOSUM test with least-squares residuals can easily be obtained from already existing tables for the moving-estimates test. Aue et al. (2009), provided the asymptotic normality of the suitably normalized stopping time of the CUSUM procedure in a similar setting. Their drawback is a strong dependence on the choice of the parameters, in particular the right choice of the window size by the statistician. Kwiatkowski et al. (1992), proposed a test of the null hypothesis that an observable series is stationary around a deterministic trend. The series is expressed as the sum of deterministic trend, random walk. The asymptotic distribution of the statistic is derived under the null and under the alternative that the series is difference-stationary. Also, Finite sample size and power are considered in a Monte Carlo experiment. Tang and Macneill (1993), shown that serial correlation can produce striking effects in distributions of change-point statistics. Failure to account for these effects is shown to invalidate change-point tests, either through increases in the type 1 error rates if low frequency spectral mass predominates in the spectrum of the noise process, or through diminution of the power of the tests when high frequency mass predominates. Lombard and Hart (1994), considered abrupt mean-change models for data with dependent, stationary, errors. No specific distributional assumptions, other than the existence and sum ability of cumulates, are made. Also, a consistency property of the least squares estimator of the change-point is derived. See also Brodsky and Darkhovsky (1993, Chapter 3), where strong mixing conditions are imposed.

Extensions of the simple change point model either allow more general change patterns or relax the independence assumption of $\left\{Z_{k}\right\}$; dependence is inevitable in the study of time series. This complicates the testing procedures. Woodward and Gray (1993), perform a test of the existence of a linear trend in autoregressive moving average models with applications to global warming. For example like the Iran rainfall data, linearity is not expected, so that a nonparametric test is desirable; Brodsky and Darkhovsky (1993), present a systematic account of nonparametric methods, and Brillinger (1989) develops a test for monotonic trends with dependent errors. The latter two papers contain many further references to test for trend. Davis, et al. (1995), considered the problem of testing whether or not a change has occurred in the parameter values and order of an autoregressive model. It is shown that if the white noise in the $A R$ model is weakly stationary with finite fourth moments, then under the null hypothesis of no change point, the normalized Gaussian likelihood ratio test statistic converges in distribution to the Gumbel extreme value distribution. Bagshaw and Richard (1997), proposed the procedures for monitoring forecast errors in order to detect changes in a time-series model. These procedures are based on likelihood ratio statistics which consist of cumulative sums. Lavielle and Moulines (1997), consider estimation and testing of multiple changes in the mean of strong mixing random processes. The assumed mean function is piecewise constant with an unknown number of pieces. Almasri (2000), used the wavelet filters for testing the trend in the presence of the fractional difference processes. The test method has been constructed using wavelet analyses which have the ability to decompose a time series into low frequencies (trend) and high frequencies (noise) components. Under the 
684 More Powerful Test for Homogeneity of Means Under an Order Restriction in Time Series with Stationary

\section{Process}

normality assumption the test statistic computed and to investigate the properties of the test statistic, empirical critical values for the test have been generated using Monte Carlo simulations. Inference based on the monotonicity assumption is discussed in Robertson et al. (1988) and Silvapulle and Sen (2005). I show that my test statistic based on isotonic regression is asymptotically more powerful than the one proposed by Brillinger (1989). Gombay and Serban (2005), proposed sequential tests for detecting an abrupt change in any parameter, or in any collection of parameters of an autoregressive time series model. These tests accommodate nuisance parameters. They are based on large sample approximations to the efficient score vector under the null hypothesis of no change and under the alternative. Gombay (2008), considered the autoregressive time series models of order $p$ and tested for change in any one of the parameters separately. He obtained the test statistics based on the efficient score vector. Also the large sample properties of the change-point estimator explored. Almasri (2010), used the approach of wavelet analysis to construct a test statistic to test for the existence of a trend in the series. He proposed a new approach for testing the presence of trend based on the periodogram of the data. Also, he studied the properties of our test statistics under different degrees of dependency and compared the results with the wavelet test with results obtained by applying the ordinary least squares method under the same conditions. Aue et al. (2012), quantified the reaction time of on-line monitoring schemes for changes in the mean based on moving sums. For an excellent review on the order restriction on mean vectors of multivariate normal populations one may refer to Silvapulle and Sen (2005), Bazyari (2012), Bazyari and Chinipardaz (2013) and Bazyari and Pesarin (2013).

In this paper, a test based on the isotonic regression is done for monotonic ordered means in time series with stationary process and short range dependent sequences errors. A test statistic is proposed using PLR approach. The test is compared this with Brillinger's (1989) method. However, there are more methods to be compared with, but it is shown that my test statistic based on isotonic regression is asymptotic more powerful than the one proposed by Brillinger (1989). To the author's knowledge, the penalized likelihood ratio test for this problem of testing with an order restriction on the unknown means in time series has not been obtained yet.

The rest of this paper is organized as follows. The test statistic is presented using PLR approach in Section 2. In Section 3, the critical values of the proposed PLR test statistic are computed by using Monte Carlo simulation for some values of sample sizes at different significance levels and the test is illustrated by real example associated with Iran rainfall data. Section 4, contains a power study and a comparison with Brillinger's (1989) test. The conclusions are summarized in Section 5. Finally, the proofs are presented in Appendix. 


\section{The test statistic}

\subsection{Preamble: Independent identically distributed normal errors}

In this subsection, we assume that $\left\{Z_{k}\right\}, k=0,1,2, \ldots$, given in (1) are independent normal random variables with mean 0 and known variance $\sigma^{2}$. Then the log likelihood function is

$$
L(\theta, \Omega)=-\frac{1}{2 \sigma^{2}} \sum_{i=1}^{n}\left(X_{i}-\theta_{i}\right)^{2}+C,
$$

where $C$ denotes a generic constant that does not depend on parameter $\theta$. The unknown parameter $\theta$ takes values in the space

$$
\Theta=\left\{\theta=\left(\theta_{1}, \ldots, \theta_{n}\right) \in R^{n}: \theta_{1} \leq \theta_{2} \leq \cdots \leq \theta_{n}\right\}
$$

Robertson et al. (1988), showed that the restricted maximum likelihood estimator of $\theta_{i}$, $i=1,2, \ldots, n$, is

$$
\min _{\mathrm{s} \geq i+1} \max _{\mathrm{r} \leq i} \frac{\sum_{\mathrm{j}=\mathrm{r}}^{\mathrm{s}-1} \overline{\mathrm{X}}_{j}}{s-r-1} .
$$

Under the null hypothesis $H_{0}$, the maximum likelihood estimator is obviously $\bar{\theta}=\bar{X}=\frac{1}{n} \sum_{i=1}^{n} X_{i}$. The exact distribution of the likelihood ratio test statistic $2\{L(\hat{\theta}, \Omega)-L(\bar{\theta}, \Omega)\}$ is given in Robertson et al. (1988). As shown below, this test statistic is affected by the so-called spiking problem in large samples, in that $\hat{\theta}_{1}$ is too small while $\hat{\theta}_{n}$ is too large. Instead of (3), we shall consider the penalized log likelihood function

$$
l_{n, r}(\theta, \Omega)=-\frac{1}{2 \sigma^{2}} \sum_{i=1}^{n}\left(X_{i}-\theta_{i}\right)^{2}-\frac{r \sqrt{n}}{\sigma^{2}}\left(\theta_{n}-\theta_{1}\right)+C,
$$

where the term $r \sqrt{n}\left(\theta_{n}-\theta_{1}\right)$, penalizes large $\left(\theta_{n}-\theta_{1}\right)$. Let $X_{1, r}=X_{1}+r \sqrt{n}$, $X_{n, r}=X_{n}-r \sqrt{n}$ and $X_{i, r}=X_{i}$ for $2 \leq i \leq n-1$. Then 
686 More Powerful Test for Homogeneity of Means Under an Order Restriction in Time Series with Stationary

$$
l_{n, r}(\theta, \Omega)=-\frac{1}{2 \sigma^{2}} \sum_{i=1}^{n}\left(X_{i, r}-\theta_{i}\right)^{2}+C
$$

maximized at

$$
\hat{\theta}_{k}=\hat{\theta}_{k, r}=\min _{\mathrm{s} \geq i+1} \max _{\mathrm{r} \leq i} \frac{X_{i, r}+\cdots+X_{j, r}}{s-r-1},
$$

which is just formula (5) with $X_{i}$ replaced by $X_{i, r}$. Furthermore, it is clear that

$$
\sum_{k=1}^{n} \hat{\theta}_{k, r}=\sum_{i=1}^{n} X_{k, r}=n \bar{X}
$$

which suggests a test statistic of the form

$$
T_{n, r}=\frac{1}{\hat{\sigma}_{n}^{2}} \sum_{k=1}^{n}\left(\hat{\theta}_{k, r}-\bar{X}\right)^{2}
$$

where $\hat{\sigma}_{n}^{2}$ is a consistence estimator of $\sigma^{2}$. If $\sigma^{2}$ is known and $\hat{\sigma}_{n}^{2}=\sigma^{2}$, then $T_{n, r}$ is the penalized $\log$ likelihood ratio statistic. The asymptotic null distribution $T_{n, r}$ is given in subsections 2.2 and 2.3.

\subsection{Short range dependent errors}

For the remainder of section 2 , let the stationary sequence $Z_{k}$, for any $k$, exhibits short range dependence in the following way. First suppose that the covariances $\gamma(k)=E\left(Z_{0} Z_{k}\right)$ are absolutely summable, i.e.

$$
\sum_{k=0}^{\infty}|\gamma(k)|<\infty
$$

Next let $T_{k}=\sum_{i=1}^{k} Z_{i}, k=0,1,2, \ldots$, and $F_{n}$ be a continuous piecewise linear function for which $F_{n}(t)=T_{k}$ for $t=\frac{k}{n}$, and suppose that 


$$
\frac{F_{n}}{\sigma \sqrt{n}} \rightarrow W
$$

where $W$ is a standard Brownian Motion and $\sigma^{2}=\gamma(0)+2 \sum_{k=1}^{\infty} \gamma(k)$.

There are many families of processes for which (10) are satisfied. If $Z_{k}$, for any $k$, is a linear process, $Z_{k}=\sum_{j=0}^{\infty} a_{j} \varepsilon_{i-j}$, say, where the $\varepsilon_{j}$ are independent and identically distributed with mean 0 and finite variance and the $a_{j}$ are absolutely summable, then (10) holds. Nonlinear process that exhibit suitable mixing conditions satisfy (10); see Peligrad's (1986) review.

If we don't assume strong mixing, sufficient conditions are that $E\left(\left|Z_{p}\right|^{k}\right)<\infty$ for some $p>2$ and that

$$
\sum_{k=1}^{\infty} k^{-\alpha} E\left\{E\left(T_{k} \mid \ldots, Z_{-1}, Z_{0}\right)^{2}\right\}<\infty
$$

for some $\alpha<2$; see Maxwell and Woodroof (2000).

The asymptotic distribution of $T_{n, r}$ is obtained in theorem 1 and 2 below for local alternatives. Suppose that

$$
\theta_{k}=\theta+\frac{\sigma}{\sqrt{n}} \phi\left(\frac{k}{n}\right), \quad k=0,1,2, \ldots
$$

where $\phi$ is a right continuous, nondecreasing function on $[0,1]$ for which

$$
\int_{0}^{1} \phi(t) d t=1
$$

The asymptotic null distribution is then a special case with $\phi=0$. Let $c=\frac{r}{\sigma}, S_{k, r}=\sum_{i=1}^{k} X_{i, r}$, $G_{n, r}(t)$, for $t \in[0,1]$, be a continuous, piecewise linear function for which $G_{n, r}\left(\frac{k}{n}\right)=\frac{S_{k, r}}{n}$ for $k=1,2, \ldots, n$, and write $S_{k}$ and $G_{n}$ for $S_{k, 0}$ and $G_{0, r}$. Define 


\section{Process}

$$
H_{n, r}(t)=\frac{\sqrt{n}}{\sigma}\left(G_{n, r}(t)-\bar{X} t\right)
$$

Let functions $L_{n, r}$ and $\Phi_{n}$ be continuous and piecewise linear for which

$$
L_{n, r}(0)=L_{n, r}(1)=0, \quad L_{n, r}\left(\frac{1}{n}\right)=L_{n, r}\left(1-\frac{1}{n}\right)=\frac{r}{\sqrt{n}}, \quad \Phi_{n}\left(\frac{k}{n}\right)=\frac{1}{n} \sum_{p=1}^{k} \phi\left(\frac{p}{n}\right)
$$

Then

$$
H_{n, r}(t)=\frac{\sqrt{n}}{\sigma}\left(F_{n}(t)-t F_{n}(1)\right)+\Phi_{n}(t)-t \Phi_{n}(1)+\frac{\sqrt{n}}{\sigma} L_{n, r}(t)
$$

Let $\Phi(t)=\int_{0}^{t} \phi(s) d s$ and define

$$
U_{c}^{\phi}(t)=W(t)-t W(1)+\Phi(t)+c I_{[0,1]}(t)
$$

Also, write $U_{c}$ for $U_{c}^{\phi}$ when $\phi \equiv 0$. Finally, if $H$ is a bounded function on [0,1], let $\tilde{H}$ denote the greatest convex minorant of $H$ and let $\tilde{h}$ denote the left-hand derivative of $\tilde{H}$. Thus $\tilde{u}_{c}^{\phi}$ is the left-hand derivative of the greatest convex minorant of $U_{c}^{\phi}$. Then $-\infty<\tilde{u}_{c}^{\phi}(0+) \leq \tilde{u}_{c}^{\phi}(1-)<\infty$ with probability 1 .

Theorem 1. If (9), (10) and (11) hold, then we have

$$
\frac{1}{\sigma^{2}} \sum_{k=1}^{n}\left(\hat{\theta}_{k, r}-\bar{X}\right)^{2} \rightarrow \int_{0}^{1}\left(\tilde{u}_{c}^{\phi}(t)\right)^{2} d t
$$

in distribution, where $c=\frac{r}{\sigma}$.

Proof. From Robertson et. al. (1988), $\hat{\theta}_{k, r}=\tilde{g}_{n, r}\left(\frac{k}{n}\right)$ for $k=1, \ldots, n$. Thus $\hat{\theta}_{k, r}-\bar{X}=\sigma \frac{\tilde{h}_{n, r}\left(\frac{k}{n}\right)}{\sqrt{n}}$ since $G_{n, r}$ and $H_{n, r}$ are linearly related, and therefore 


$$
\frac{1}{\sigma^{2}} \sum_{k=1}^{n}\left(\hat{\theta}_{k, r}-\bar{X}\right)^{2}=\int_{0}^{1}\left(\tilde{h}_{n, r}(t)\right)^{2} d t
$$

Thus it is necessary to show that the right hand side of (15) converges in distribution to the right hand side of (14). This seems plausible in view of (10) and (12). Details may be found in the Appendix.

Remark 1. If $c=0$, then the right hand side (14) in infinity with probability 1 ; see Groeneboom and Pyke (1983). This is the spiking problem mentioned above. The left hand side of (14) does not have a nondegenerate asymptotic under null hypothesis.

Remark 2. In the context of classical change point analysis, there is a similar problem. Suppose that we want to test the following hypothesis

$$
H_{1}^{\prime \prime}: \theta_{1}=\theta_{2}=\cdots=\theta_{v}<\theta_{v+1}=\cdots=\theta_{n} .
$$

Let $\bar{X}=\frac{1}{r} \sum_{i=1}^{r} X_{i}$ and $\bar{X}_{n-r}^{\prime}=\frac{1}{n-r} \sum_{i=r+1}^{n} X_{i}$. It is mentioned in section 2.4 of Bhatacharya (1994) that the test statistic based on the likelihood ratio

$$
\max _{1 \leq r \leq n-1}\left(\frac{1}{r}+(n-r)^{-1}\right)^{-\frac{1}{2}}\left(\bar{X}_{n-r}^{\prime}-\bar{X}\right),
$$

does not have an asymptotic distribution, since it becomes unstable at the ends.

\subsection{Estimation of parameter $\sigma^{2}$}

If the $Z_{i}$, for any $i$, were observed, then the covariance function $\gamma(k)=E\left(Z_{0} Z_{k}\right)$ could be estimated by the sample covarinces $\bar{\gamma}_{n}(k)=\frac{1}{n} \sum_{i=1}^{n-k} Z_{i} Z_{i+k}$. Then $\sigma^{2}$ could be estimated by the lag window estimators

$$
\bar{\sigma}_{n}^{2}=\bar{\gamma}_{n}(0)+2 \sum_{k=1}^{m_{n}} w\left(\frac{k}{m_{n}}\right) \bar{\gamma}_{n}(k),
$$


where the lag window $w$ satisfies $w(0)=1, w(x)=0$ for $|x| \geq 1$ and $|w(x)| \leq 1$ for all $x$; see Brockwell and davis (1991, equation (10.4.8)). Under conditions of their Theorem 10.4.1, $\bar{\sigma}_{n}^{2}$ is a consistent estimator of $\sigma^{2}$ provided that $m_{n} \rightarrow \infty$ and $m_{n}=o(n)$.

Recall that the unpenalized isotonic regression estimator $\hat{\theta}$ is given by (5). Hence for any $k$, the detrended process is $\hat{Z}_{k}=X_{k}-\hat{\theta}_{k}$. Note that the sum of residuals $\sum_{k=1}^{n} \hat{Z}_{k}=0$. Let

$$
\hat{\gamma}_{n}(k)=\frac{1}{n} \sum_{i=1}^{n-i} \hat{Z}_{i} \hat{Z}_{i+k}
$$

and consider estimators of the form

$$
\hat{\sigma}_{n}^{2}=\hat{\gamma}_{n}(0)+2 \sum_{k=1}^{m_{n}} w\left(\frac{k}{m_{n}}\right) \hat{\gamma}_{n}(k),
$$

where $m_{n} \rightarrow \infty$ and $m_{n}=o(n)$.

Theorem 2. Assume that the $Z_{k}$ satisfy (9) and (10), and let $\hat{\theta}_{k}$ is of the form (11). If $m_{n} \rightarrow \infty$, $m_{n}=o(\sqrt{n})$ and $\hat{\sigma}_{n}^{2}$ is a consistent estimator of $\sigma^{2}$, then $\hat{\sigma}_{n}^{2}$ converges to $\sigma^{2}$ in probability.

Proof. In view of (16), it suffices to show that

$$
\max _{0 \leq k \leq n-1}\left|\hat{\gamma}_{n}(k)-\bar{\gamma}_{n}(k)\right|=O_{p}\left(n^{-\frac{1}{2}}\right),
$$

see the Appendix for details.

Meyer and Woodroofe (2000), have established the asymptotic normality of $\hat{\gamma}_{n}(0)$ when the errors are independent and normally distributed. In the remainder of the paper $T_{n, r}$ is defined by (8) with $\hat{\sigma}_{n}^{2}$ dfined by (17).

Corollary 1. Suppose that $H_{0}$ is true, then under the conditions of theorem 2 


$$
T_{n, r} \rightarrow \int_{0}^{1}\left(\tilde{u}_{c}(t)\right)^{2} d t
$$

in distribution, where $c=\frac{r}{\sigma}$. Note that in practice, $r$ must be $c \hat{\sigma}_{n}$. This does not affect the asymptotic distribution.

\section{Applications of test}

\subsection{Estimation of the asymptotic null distribution $T_{n, r}$}

The asymptotic null distribution of $T_{n, r}$ given in (8) is complicated, but the critical points can be computed by Monte Carlo simulation method. The complete source programs are written in $R$ software. We simulated 10000 Brownian motions and computed the right hand side of (19) for each of them. The asymptotic null distribution was estimated from these values. Brownian motion was simulated by generating standard normal variates $Z_{k}$ and forming piecewise linear functions with value $\frac{\left(Z_{1}+\cdots+Z_{k}\right)}{\sqrt{n}}$ at $t=\frac{k}{n}$ for $k=1, \ldots, n$. This simulation is done for some of the different values of $n$. The number of grid points was $n=20,50,100,200,500,1000,2000$. The critical values $t_{c}(\alpha)$ are given in Table 1 for some of the different values of $\alpha$ and $c$, $\alpha=0.05,0.01$ and $c=0.05,0.1,0.15$.

Table 1. Critical values $t_{c}(\alpha)$ for $\alpha=0.05,0.01$ and $c=0.05,0.1,0.15$

\begin{tabular}{c|cc|cc|cc}
\hline$n$ & \multicolumn{2}{|c|}{$c=0.05$} & \multicolumn{2}{c|}{$c=0.01$} & \multicolumn{2}{c}{$c=0.15$} \\
\hline & 0.05 & 0.01 & 0.05 & 0.01 & 0.05 & 0.01 \\
\hline 20 & 7.01 & 10.76 & 6.10 & 9.62 & 5.32 & 8.65 \\
50 & 8.16 & 12.27 & 6.99 & 10.68 & 6.01 & 9.47 \\
100 & 9.13 & 13.36 & 7.70 & 11.58 & 6.59 & 10.27 \\
200 & 9.82 & 14.30 & 8.17 & 12.23 & 6.95 & 10.74 \\
500 & 10.49 & 15.30 & 8.58 & 12.85 & 7.19 & 11.27 \\
1000 & 10.70 & 15.25 & 8.70 & 12.98 & 7.34 & 11.39 \\
2000 & 11.02 & 15.56 & 8.88 & 13.03 & 7.45 & 11.35 \\
\hline
\end{tabular}


In the following example, we used the values $c=0.15$ and $m_{n}=n^{\frac{1}{3}}$, and the truncated window, namely $w(x)=1$, for $|x| \leq 1$ and $w(x)=0$ otherwise. These values performed well in simulations and 0.15 was chosen for $c$ after comparing power curves for selected alternatives.

\subsection{Iran rainfall data}

Figure 1 shows the volume of yearly rainfall in Iran from 1898 to 2010 along with the penalized and unpenalized isotonic regression functions. There is a mild spiking problem: the year 1898 has a low value. The spiking problem is clearly suppressed in the penalized isotonic regression. From an autocorrelation plot, the residuals appear to be short range dependent. Only the eighth of the first twenty autocorrelations is significant at the 0.05 level. From (8) and (17), $\hat{\sigma}^{2}=253.57$ and $T_{n, r}=17.95$, which is significant at the 0.01 level since the 0.99 quantile is between 10.27 and 10.74 from Table 1 . Thus the apparent increase in the mean is highly significant.



Figure 1. Iran rainfall Data: yearly rainfall (millimeters) in Iran from 1898 to 2010 with its penalized and unpenalized isotonic regression functions

From the penalized isotonic regression in Figure 1, we can see there is a large jump around the years 1970-1971. This observation justifies the belief of meteorologist that the increase might be caused by construction of a dam. The mean rainfall volume is 76.34 before 1971 and 90.11 after 1971. 
Now let us consider Brillinger's (1989) test. If we use the estimated variance $\hat{\sigma}^{2}=253.57$, the test statistic, see Brillinger (1989, equation (2.3)), is

$$
\frac{\sum_{k=1}^{n} v(k) X_{k}}{\hat{\sigma}\left\{\sum_{k=1}^{n} v^{2}(k)\right\}^{\frac{1}{2}}}=\frac{41.0621}{29.1645}=1.4079
$$

where $v(t)=\left((t-1)\left(1-\left(\frac{t}{n}-\frac{1}{n}\right)\right)\right)^{\frac{1}{2}}-\left(t\left(1-\frac{t}{n}\right)\right)^{\frac{1}{2}}, \quad(1 \leq t \leq n)$.

Then the $p$-value is $P_{r}(Z>1.4079)=0.0796$, where $Z$ is standard normal. Hence, at the 0.05 level of significance, Brillinger's (1989) test is unable to detect the trend. Section 4, contains some power comparisons.

\section{Power study of test statistic}

In this section, the power of our test statistic $T_{n, r}$ is compared to that of the proposed by Brillinger (1989). Suppose that the trend has the form

$$
\theta_{k}=\frac{\delta}{\sqrt{n}} \phi\left(\frac{k}{n}\right) \quad(\delta>0), \quad k=1,2, \ldots, n
$$

where $\phi$ is a nondecreasing function with $\int_{0}^{1} \phi(t) d t=0$. The test developed by Brillinger (1989) is unable to detect the trend (20) with $\delta=\delta_{n} \rightarrow \infty$ and $\delta_{n}=o\left((\log n)^{\frac{1}{2}}\right)$; see Brillinger's (1989) equation (3.2). On the other hand, suppose that $\phi$ is not identically 0 , then by theorem 1 , the asymptotic power of test based on (8) is given by

$$
P_{r}\left[\int_{0}^{1}\left(\tilde{u}_{c}^{\eta \phi}(t)\right)^{2} d t>t_{c}(\alpha)\right]
$$

where $\eta=\frac{\delta}{\sigma}$. 
694 More Powerful Test for Homogeneity of Means Under an Order Restriction in Time Series with Stationary

\section{Process}

Figure 2(a) presents a simulated power study, when $Z_{i}$ are independent and identically distributed as standard normal, $\theta_{k}=-\frac{1}{2}$, for $1 \leq k \leq 100, \theta_{k}=\frac{1}{2}$, for $101 \leq k \leq 200$ and $\delta=\frac{k}{100}$, for $k=0,1, \ldots, 99$. From the plot, we see that the testing procedure based on isotonic regression is more powerful than Brillinger's (1989) test. In Figure 2(b), the mean function is linear: $\theta(k)=\frac{k}{200}-0.5$, for $1 \leq k \leq 200$, and $\delta=\frac{k}{100}$, for $k=0,1, \ldots, 99$. Similar conclusions hold.


Figure 2. Power curves for the uniformly most powerful test (dot-dashed lines), the isotonic regression test and Brillinger's (1989) test (dotted) for (a) step function with independently distributed $N(0,1)$ errors, and

(b) linear function with independently distributed $N(0,1)$ errors

\section{Conclusions}

The present article considers an approach to testing homogeneity of several means under an order restriction in time series with stationary process and short range dependent sequences errors. This approach is based on penalized likelihood ratio and the test statistic obtained and its critical values computed by using Monte Carlo simulation for some values of sample sizes at different significance levels. To show the application of the proposed test the real dataset contains monthly Iran rainfall records collected from 1898 to 2010. The volume of yearly rainfall in these years shown in Figure 1 along with the penalized and unpenalized isotonic regression functions. In the year 1898 there was a low value of mild spiking problem. The value of test statistic computed along with the data which is significant at the 0.1 level. Also, from the Figure 2, it was clear that the testing procedure based on isotonic regression is more powerful than Brillinger's (1989) test. 


\section{Appendix}

Proof of Theorem 1. The proof uses strong approximation. If the probability space sufficiently rich, then there are versions $F_{n}^{\prime}$ of $F_{n}$ and Brownian motions $W_{n}$ such that

$$
\sup _{0 \leq t \leq 1}\left|(\sigma \sqrt{n})^{-1} F_{n}^{\prime}-W_{n}(t)\right| \rightarrow 0
$$

in probability, and there is no loss of generality in supposing that $F_{n}^{\prime}=F_{n}$ for the purposes of proving Theorem 1.

Recall the definitions (12) and (13) of $H_{n}$ and $U_{c}^{\phi}$ and relation (15), and define $U_{n, c}^{\phi}$ by (13) with $W$. Then it suffices show that

$$
\int_{0}^{1}\left(\tilde{h}_{n}(t)\right)^{2} d t-\int_{0}^{1}\left(\tilde{u}_{n, c}^{\phi}(t)\right)^{2} d t \rightarrow 0
$$

in probability as $n \rightarrow \infty$, since $\int_{0}^{1}\left(\tilde{u}_{n, c}^{\phi}(t)\right)^{2} d t$ has the same distribution as $\int_{0}^{1}\left(\tilde{u}_{c}^{\phi}(t)\right)^{2} d t$ for all $n$.

Lemma 1. The functions $\tilde{h}_{n}(0+)$ and $\tilde{h}_{n}(1-)$ are stochastically bounded.

Proof. Consider that

$$
\begin{aligned}
P\left(\tilde{h}_{n}(0+)<-a\right) & =P\left(\sqrt{n} \min _{k \leq n} \frac{S_{k, r}}{\sigma k}<-a\right) \\
& \leq P\left(\max _{k \leq n \sigma}\left|\frac{S_{k}}{\sigma(n \delta)^{\frac{1}{2}}}\right|>\frac{c}{\sqrt{\delta}}\right)+P\left(\max _{n \delta \leq k \leq n}\left|\frac{S_{k}}{\sigma \sqrt{n}}\right|>a \sqrt{\delta}\right),
\end{aligned}
$$

where $\delta=\frac{1}{\sqrt{a}}$. Since $\max _{k \leq n}\left|\frac{S_{k}}{\sqrt{n}}\right|$ is stochastically bounded, it then follows that $P\left(\tilde{h}_{n}(0+)<-a\right) \rightarrow 0$, as first $n \rightarrow \infty$ and then $a \rightarrow \infty$. This establish the stochastic boundedness of $\tilde{h}_{n}(0+)$. The right endpoint may be handled similarly.

Now, to prove (18), the also following lemma is needed. 
696 More Powerful Test for Homogeneity of Means Under an Order Restriction in Time Series with Stationary

\section{Process}

Lemma 2. If $-\infty<a_{1} \leq a_{2} \leq \cdots \leq a_{n}<\infty$ and $-\infty<b_{1}<b_{2}<\cdots<b_{n}<\infty$, then

$$
\left|\sum_{j=1}^{n} a_{j} b_{j}\right| \leq\left(\left|a_{n}\right|+\left(a_{n}-a_{1}\right)\right) \max _{j \leq n}\left|\sum_{i=1}^{j} b_{i}\right| .
$$

Proof. Let for any $k, B_{k}=b_{1}+\cdots+b_{k}$. Then the term on the left hand side is

$$
\left|a_{n} B_{n}-\sum_{j=2}^{n}\left(a_{j}-a_{j-1}\right) B_{j-1}\right| \leq\left(\left|a_{n}\right|+\left(a_{n}-a_{1}\right)\right) \max _{j \leq n}\left|B_{j}\right|,
$$

as asserted.

To prove (18), now write

$$
\hat{\gamma}_{n}(k)-\bar{\gamma}_{n}(k)=\frac{1}{n} \sum_{i=1}^{n-k}\left(\hat{Z}_{i} \hat{Z}_{i+k}-Z_{i} Z_{i+k}\right)=I_{n}(k)+I I_{n}(k)+I I I_{n}(k),
$$

where

$$
I_{n}(k)=\frac{1}{n} \sum_{i=1}^{n-k}\left(\theta_{i}-\hat{\theta}_{i}\right) Z_{i+k}, \quad I I_{n}(k)=\frac{1}{n} \sum_{i=1}^{n-k}\left(\theta_{i+k}-\hat{\theta}_{i+k}\right) Z_{i},
$$

and

$$
I I I_{n}(k)=\frac{1}{n} \sum_{i=1}^{n-k}\left(\theta_{i}-\hat{\theta}_{i}\right)\left(\theta_{i+k}-\hat{\theta}_{i+k}\right) .
$$

here

$$
I_{n}(k) \leq 2\left(\left|\theta_{n}\right|+\left|\theta_{1}\right|+\left|\hat{\theta}_{1}\right|+\left|\hat{\theta}_{n}\right|\right) \max _{j \leq n} \frac{\left|Z_{1}+Z_{2}+\cdots+Z_{j}\right|}{n},
$$

Also, $\hat{\theta}_{n}$ and $\hat{\theta}_{1}$ are stochastically bounded by Lemma 1 . Thus $\max _{k \leq n}\left|I_{n}(k)\right|=O_{p}\left(n^{-\frac{1}{2}}\right)$, and similarly $\max _{k \leq n}\left|I I_{n}(k)\right|=O_{p}\left(n^{-\frac{1}{2}}\right)$. For $I I I_{n}(k)$, let $\bar{\theta}=\frac{1}{n} \sum_{k=1}^{n} \theta_{k}$. Then we get 


$$
\sum_{i=1}^{n}\left(\hat{\theta}_{i}-\theta_{i}\right) \leq 4\left(\sum_{i=1}^{n}\left(\hat{\theta}_{i}-\bar{X}_{n}\right)^{2}+\sum_{i=1}^{n}\left(\theta_{i}-\bar{\theta}\right)^{2}+n\left(\bar{X}_{n}-\bar{\theta}\right)^{2}\right)
$$

which is stochastically bounded by theorem 1 and formulas (9) and (11). Also $\max _{k \leq n}\left|I I I_{n}(k)\right|=O_{p}\left(\frac{1}{n}\right)$, then follows from Schwart's inequality.

\section{Acknowledgment}

The author is deeply grateful to Editor in chief and the referees for valuable comments and suggestions.

\section{References}

[1] Almasri, A. (2000). Some Aspects of Wavelet Analysis in Time Series, Research Report Department of Statistics, Goteborg University, Sweden.

[2] Almasri, A. (2010). Tests for trend: A simulation study, Communications in StatisticsSimulation and Computation, 39(3), 598-611.

[3] Aue, A., Horváth, L., and. Reimherr, M. L. (2009). Delay times of sequential procedures for multiple time series regression models, Journal of Econometrics, 149(2),174-190.

[4] Aue, A., Hörmann, S., Horváth, L. and Hušková, M. (2012). Sequential Stability Tests for Functional Linear Models, Preprint, University of California Davis, Université Libre de Bruxelles, University of Utah, Charles University in Prague.

[5] Bagshaw, M. and Richard, A. J. (1997). Sequential procedures for detecting parameter changes in a time-series model, Journal of the American Statistical Association, 72(359), 593-597.

[6] Bai, J. (1997). Estimation of a change point in multiple regression models, Review of Economics and Statistics, 551-563.

[7] Bazyari, A. (2012). On the computation of some properties of testing homogeneity of multivariate normal mean vectors against an order restriction. METRON, International Journal of Statistics, 70 (1), 71-88.

[8] Bazyari, A. and Pesarin, F. (2013). Parametric and permutation testing for multivariate monotonic alternatives, Statistics and Computing, 23 (5), 639-652. 
698 More Powerful Test for Homogeneity of Means Under an Order Restriction in Time Series with Stationary

\section{Process}

[9] Bazyari, A. and Chinipardaz, R. (2013). Upper bound for p-value of the test of multivariate normal ordered mean vectors against all alternatives, Communications in Statistics-Theory and Methods, 42: 1748-1758.

[10] Bhattacharya, P. K. (1994). Some aspects of change point analysis. Change point problems, Institute of Mathematical Statistics, Hayward, CA, 28-56.

[11] Brillinger, D. R. (1989). Consistent detection of a monotonic trend superposed on a stationary time series, Biometrika, 76, 23-30.

[12] Brockwell, P. J. and Davis, R. A. (1991). Time series: Theory and Methods, New York, springer.

[13] Brodsky, B. F. and Darkhovsky, B. S. (1993). Nonparametric Methods in Change Point Problems, Dordrecht, Kluwer.

[14] Chu, C. S. J., Hornik, K., and Kuan, C. M. (1995). MOSUM tests for parameter constancy, Biometrika, 82(3), 603-617.

[15] Csörgo, M. and Horváth, L. (1997). Limit Theorems in Change-Point Analysis, Wiley, New York.

[16] Darkhovsky, B. S. (1994). Nonparametric method in change point problem: a general change approach and some concrete algorithms, In Change-point Problems, Ed, E. Carlstein, H. Muller and D. Siegmend, pp. 99-107, Hayward, CA: Inst. Math. Statist.

[17] Davis, et al. (1995). Testing for a change in the parameter values and order of an autoregressive model, The Annals of Statistics, 23(1), 282-304.

[18] Eggermont, P. P and LaRiccia, V. N. (2009). Maximum Penalized Likelihood Estimation: Volume II: Regression, Springer, New York.

[19] Gombay, E. and Serban, D. (2005). Monitoring parameter change in AR(p) time series models, Statistics Centre Technical Reports 05.04, The University of Alberta, Edmonton, Canada.

[20] Gombay, E. (2008). Change detection in autoregressive time series, Journal of Multivariate Analysis, 99(3), 451-464.

[21] Groeneboom, P. and Pyke, R. (1983). Asymptotic normality of statistics based on the convex minorants of empirical distribution functions, The Annals of Probability, 11, 328-345.

[22] Horváth, L., Hušková, M., Kokoszka, P., and Steinebach, J. (2004). Monitoring changes in linear models, Journal of Statistical Planning and Inference, 126(1), 225-251. 
[23] Kwiatkowski, D., Phillips, P.C.B., Schmidt, P., and Shin, Y. (1992). Testing the null hypothesis of stationarity against the alternative of a unit root, Journal of Econometrics, 54, 159-178, North-Holland.

[24] Lavielle, M. and Moulines, E. (1997). Detection de ruptures multiples dans la moyenne d'un processus aleatoire, C. R. Acad, Sci. Paris Ser. I Math. 324, 239-243.

[25] Lombard, F. and Hart, J. D. (1994). The analysis of change-point data with dependent errors, Change-point problems, Institute of Mathematical Statistics, 23, 194-209,

[26] Maxwell, M. and Woodroofe, M. (2000). Central limit theorems for additive functions of Markov chains, The Annals of Probability, 28, 713-724.

[27] Meyer, M. and Woodroofe, M. (2000). On the degrees of freedom in shape-restricted regression, The Annals of Statistics, 28, $1083-1104$.

[28] Peligrad, M. (1986). Recent advances in the central limit theorem and its weak invariance principle for mixing sequence of random variance (a survey). In dependence in Probability and Statistics: A survey of Recent Results, Ed. E. Eberlein and M. Taqqu, pp. 193-224. Boston, MA: Birkhauser.

[29] Perron, P. (2006). Dealing with structural breaks. Palgrave Handbook of Econometrics, 1, 278-352.

[30] Picard, D. (1985). Testing and estimating change-points in time series, Advances in Applied Probability, 17, 841-867.

[31] Robertson, T., Wright, F. T. and Dykstra, R. L. (1988). Order Restricted Statistical Inference, New York, John Wiley.

[32] Shaban, S. A. (1980). Change point problem and two-phase regression: A annotated bibliography, International Statistical Review, 48, 83-93.

[33] Siegmund, D. (1986). Boundary crossing probability and statistical applications. The Annals of Statistics, 14, 361-404.

[34] Silvapulle M. J. and Sen P. K. (2005). Constrained Statistical Inference: Inequality, Order, and Shape Restrictions, New York, John Wiley.

[35] Tang, S. M. and MacNeill, I. B. (1993). The effect of serial correlation on tests for parameter change at unknown time, The Annals of Statistics, 21, 552-575.

[36] Woodward, W. A. and Gray, H. L. (1993). Global warming and the problem of testing for trend in time series data, Journal of Climate, 6, 953-052. 
700 More Powerful Test for Homogeneity of Means Under an Order Restriction in Time Series with Stationary

\section{Process}

Received November 15, 2015; accepted January 09, 2016.

Abouzar Bazyari

Department of Statistics, Persian Gulf University, Bushehr, Iran

Email: ab_bazyari@yahoo.com 